http:// bjas.bu.edu.eg

\title{
Skin hypersensitivity test against nasal bots infestations in donkeys and camels
}

A.A.Balegh ${ }^{1}$, L.M.El-akabawy ${ }^{2}$, M.Y.Ramadan ${ }^{2}$, S.F.Omar ${ }^{1}$ and R.S.El-madawy ${ }^{2}$

${ }^{1}$ Parasitology Dept., Animal Health Research Institute, Dokki, Giza, Egypt

${ }^{2}$ Parasitology Dept., Faculty of Veterinary Medicine, Banha Univ., Banha, Egypt

E-Mail: aliaabaleg@yahoo.com

\begin{abstract}
The objective of this study was assessing the Intradermal test (IDT) in the diagnosis of both cephalopinosis and rhinoestrosis. 6 males one-humped dromedary camels (Camelus dromedarius) admitted to camel hosting house of El-Basateen slaughter house, beside, 12 female Egyptian donkeys (Equus asinus) admitted to Giza zoo abattoir from Bani-suef were subjected to an intradermal injection of $0.5 \mathrm{ml}$ of PBS PH (7.2) at one side of the neck and this served as negative control; on the other side of the neck each animal received 3 intradermal injections of 3 different protein concentrations $(0.5 \mathrm{mg} / \mathrm{ml}, 1 \mathrm{mg} / \mathrm{ml}$ and $1.5 \mathrm{mg} / \mathrm{ml})$ for each antigen $\left(1^{\text {st }}\right.$ larval instars crude extract and salivary gland extract of Cephalopina titillator larvae, and, Excretory secretory product (ESP), Salivary gland extract, Mid-gut extract and Mixed crude extract of Rhinoestrus spp. larvae). The results of the IDT were determined through measuring the diameter of the resulting wheals using a scale bar and by detection of presence or absence of skin reactions. The swelling size in the skin was increased with increasing the concentration of the injected antigen. Most animals showed skin reactions after $30 \mathrm{~min}$. The results signified the critical role of IDT in early diagnosis of cephalopinosis and rhinoestrosis.
\end{abstract}

Keywords: Cephalopinosis, Rhinoestrosis, Intradermal test, Diagnosis.

\section{Introduction}

Cephalopina titillator (Diptera, Oestridae) is an obligate parasite of domesticated camel $[16,2]$. It causes cephalopinosis that considered as a prevalent parasitic problem in Egypt $[1,16,5]$.

Rhinoestrosis is a parasitic disease caused by larvae of Rhinoestrus spp. (Diptera, Oestridae), which localize in nasal cavities, sinuses and pharynx of horses, donkeys and zebras [19]. The presence of larvae of Cephalopina titillator and Rhinoestrus spp. induces local inflammation and the infection is characterized by clinical designations of varying intensity and astringency, ranging from inflammation to dyspnea, sneezing and cough [10]. Moreover, lesions of the upper respiratory tract, lungs and damage of the olfactory nerves were reported [9].

Although $C$. titillator prevails among camel spp., their importance is still underestimation [14]. In contrast to numerous Oestrids Cephalopina adult fly don't panic or fright the camels, and great numbers are frequently noticed resting on the camels' heads and around the nostrils. Diagnosis of C. titillator in the antecedent studies depended mainly on the post-mortem examinations [3, 13].

Diagnosis of equine rhinoestrosis in live animal is hard even when using endoscope because; the larvae when present are very difficult to be encountered in the pharynx behind the velaum palatinum.

There have been few studies on the use of skin hypersensitivity tests for diagnosis of Myasis producing flies $[4,6-8,11]$. The present work is the first study for assessing the Intradermal test in the diagnosis of both cephalopinosis and rhinoestrosis.

\section{Materials and methods}

Intradermal test (IDT) was carried out according to [12] and [4].

\subsection{Animals}

In June, 6 males one-humped dromedary camels (Camelus dromedarius) of 7-8years old, admitted to camel hosting house of El-Basateen slaughter house and showed absence of any clinical signs to cephalopinosis. Also, in May, 12 female Egyptian donkeys (Equus asinus) of different ages (21-28 years) were admitted to Giza zoo abattoir from Bani-suef. Donkeys were free from any clinical signs of rhinoestrosis. The camels were divided into 2 groups each containing 3 animals. The donkeys were divided to 4 groups each containing 3 animals.

\subsection{Experiment design}

An area of $30 \mathrm{~cm} \times 15 \mathrm{~cm}$ at the posterior third of the dorsolateral cervical region of the neck was clipped from both sides and disinfected with ethyl alcohol $70 \%$.

Each animal received an intradermal injection of $0.5 \mathrm{ml}$ of PBS pH (7.2) at one side of the neck and this served as negative control; on the other side of the neck each animal received 3 intradermal injections of 3 different protein concentrations $(0.5 \mathrm{mg} / \mathrm{ml}, 1 \mathrm{mg} / \mathrm{ml}$ and $1.5 \mathrm{mg} / \mathrm{ml})$ for each antigen using $1.5 \mathrm{cc}$ syringes and 21 gauge needles.

The result of the IDT was determined through measuring the diameter of the resulting wheals using a scale bar and by detection of presence or absence of skin reactions (erythrematous swellings or ulcers) at $1 / 2 \mathrm{hr}, 1 \mathrm{hr}, 24 \mathrm{hr}, 48 \mathrm{hr}$ and $72 \mathrm{hr}$ post injection. Confirmation of the IDT was done through postmortem examination of slaughtered animals' heads.

2.3 Statistical analysis 
The Statistical analysis was carried out using ANOVA with five factors under significance level of 0.05 for the whole results using SPSS (ver. 22). Data were treated as complete randomization design according to [17]. Multiple comparisons were carried out applying LSD.

\section{Results and discussion}

Data recorded in table (1) and (3) indicated that the intradermal injection of different $C$. titillator (L1CE and SG) and Rhinoestrus spp. larval antigens (ESP, SG, MG, MixCE) produced edematous skin reactions of varying intensity according to the antigen type and the induration's size of the skin increased with increasing the concentration of the injected antigen. Also, there was significant difference in the skin reactions produced by different antigen concentrations $(p<0.05)$ in the control and the tested animals.

Concerning the effect of different $C$. titillator larval antigens on the cellular immunity of camels, data obtained from Table $(1,2)$ Fig (1) Plate $(1,2)$ proved that the skin edema and swelling of the skin were significantly enhanced with increasing the concentration of the injected antigen. The skin edematous swelling was significantly increased after $1 \mathrm{hr}$ then began to drop off after $24 \mathrm{hrs}$ to reach its low size after $72 \mathrm{hr}$.

Unlike the succeeding results of donkeys, the first larval crude extract of $C$. titillator presented the highest cellular response with a severe increase in the skin edema. Erythematous reaction was difficult to be noticed because of the black color of the camel's skin.

Concerning the effect of different Rhinoestrus spp. larval antigens on the cellular immunity of donkeys data obtained from Table $(3,4)$ Fig $(2)$ Plate $(3,4,5$ and 6$)$ revealed that the skin induration was produced after $30 \mathrm{~min}$. of injection of all the used antigens to be more palpable after $1 \mathrm{hr}$. However, after $24 \mathrm{hr}$ the skin induration's size began to diminish gradually to reach a low size after $72 \mathrm{hr}$ except for the salivary gland antigen and the mid-gut antigen in two animals, where the intense inflammatory skin reaction was clearly visible and reaches its maximum response after $24 \mathrm{hr}$ then began to gradually subside. On the other hand, injection of PBS in the control negative cases resulted in relatively small size indurations in all animals that soon completely subsided after $1 / 2$ hrs. reaction than the other concentrations. There was a significant increase in the induration's size and strong skin reactions after $1 \mathrm{hr}$ and $24 \mathrm{hr}$ than after $30 \mathrm{~min}$., $48 \mathrm{hr}$ and $72 \mathrm{hr}$. There was significant difference among all antigens, the strongest reaction was to the $\mathrm{SG}$ extract antigen followed by ESP antigen. On the other hand, there was no significant difference between $\mathrm{MG}$ antigen and MixCE antigen.

Intradermal test was confirmed by careful postmortem examination of the tested donkeys through detection of Rhinoestrus spp. larvae in their heads. The post-mortem examination of the tested donkeys showed that all the injected animal's heads had more than one Rhinoestrus spp. $1^{\text {st }}$ stage larvae beside one animal had only one L3.

Our results illustrated that the cellular immunity response after intradermal injection of different larval antigens either for Rhinoestrus spp. or for $C$. titillator in donkeys and camels respectively, was clearly palpable after $1 \mathrm{hr}$ that indicated presence of an immediate type of hypersensitivity reactions. This is the first record of such type of hypersensitivity in donkeys and camels.

These results were parallel to those obtained by other scientists on Oestrus ovis of sheep [4, 7] and on Hypoderma lineatum in cattle [11, 15].

On the other hand there was late type of hypersensitivity due to involvement of allergenspecific T-cells that presented by salivary gland antigen and to a lesser extent by mid-gut antigens after $24 \mathrm{hr}$ and this was analogous to that obtained by [12] where they explained presence of late response by presence of irritant substances in saliva (of Culicoides) that caused non-allergen mediated inflammation and these substances were able to generate edema and erythema in the laboratory animals and humans.

\section{Conclusion}

The results recorded in our study showed that the highest response to intradermal test was after $30 \mathrm{~min}-1 \mathrm{hr}$ so it magnified the critical role of intradermal test in early diagnosis of rhinoestrosis and cephalopinosis.

\section{Acknowledgements}

I would like to express my immense appreciations to all veterinarians and workers of El-Basateen hosting house and Giza zoo for their help, encouragement and good hospitality.

There was a significant effect $(p<0.05)$ to the concentration of $1.5 \mathrm{mg}$ of all antigens on the skin 
Table (1) Skin reaction measurements (Inch) during different periods by different Cephalopina titillator larval antigens concentrations

\begin{tabular}{|c|c|c|c|c|c|c|c|c|c|}
\hline \multirow{3}{*}{ Time(P.I) } & \multirow{3}{*}{$\begin{array}{l}\text { Animal } \\
\text { number }\end{array}$} & \multicolumn{8}{|c|}{ Antigen type } \\
\hline & & \multicolumn{4}{|c|}{ First larval crude extracrt } & \multicolumn{4}{|c|}{ Salivary gland extract } \\
\hline & & $\begin{array}{c}\text { Control } \\
\text { negative }\end{array}$ & $0.5 \mathrm{mg}$ & $1 \mathrm{mg}$ & $1.5 \mathrm{mg}$ & $\begin{array}{c}\text { Control } \\
\text { negative }\end{array}$ & $0.5 \mathrm{mg}$ & $1 \mathrm{mg}$ & $1.5 \mathrm{mg}$ \\
\hline \multirow{3}{*}{$30 \mathrm{~min}}$. & 1 & 0 & 6 & 6.4 & 7.1 & 0 & 4 & 4.5 & 4.9 \\
\hline & 2 & 0 & 3.2 & 4 & 6 & 0 & 3.5 & 4.6 & 5.5 \\
\hline & 3 & 0 & 6.5 & 7.8 & 9.5 & 0 & 5 & 5.9 & 6 \\
\hline \multirow{3}{*}{$1 \mathrm{hr}$} & 1 & 0 & 7.4 & 6.9 & 7.6 & 0 & 5.1 & 6 & 6.5 \\
\hline & 2 & 0 & 5 & 5.6 & 7.3 & 0 & 3.6 & 4.8 & 5.9 \\
\hline & 3 & 0 & 6.6 & 8.1 & 10 & 0 & 6.9 & 7.3 & 7.8 \\
\hline \multirow{3}{*}{$24 \mathrm{hr}$} & 1 & 0 & 4.6 & 5.5 & 6 & 0 & 3.1 & 4.3 & 4.8 \\
\hline & 2 & 0 & 3.1 & 3.8 & 5.9 & 0 & 3.2 & 3.4 & 3.5 \\
\hline & 3 & 0 & 5.5 & 5.9 & 6 & 0 & 3.8 & 4.5 & 5.2 \\
\hline \multirow{3}{*}{$48 \mathrm{hr}$} & 1 & 0 & 4.2 & 4.5 & 5 & 0 & 3.1 & 4.2 & 4.5 \\
\hline & 2 & 0 & 3 & 3.7 & 5.9 & 0 & 2.8 & 2.9 & 3 \\
\hline & 3 & 0 & 4.6 & 5.5 & 6 & 0 & 3.5 & 3.9 & 4.5 \\
\hline \multirow{3}{*}{$72 \mathrm{hr}$} & 1 & 0 & 4 & 4.3 & 4.9 & 0 & 3 & 3.7 & 4 \\
\hline & 2 & 0 & 2.6 & 3.5 & 4.8 & 0 & 2.5 & 2.9 & 3 \\
\hline & 3 & 0 & 3 & 3.5 & 4.3 & 0 & 3.4 & 3.8 & 3.9 \\
\hline
\end{tabular}

-P.I.: post injection

Table (2) Mean size of skin reaction (Inch) using different $C$. titillator larval antigen concentrations at different period of time

\begin{tabular}{|c|c|c|c|c|c|c|c|}
\hline \multirow{2}{*}{$\begin{array}{c}\text { Antigen } \\
\text { type }\end{array}$} & \multirow{2}{*}{$\begin{array}{c}\text { Concentration } \\
\text { (mg) }\end{array}$} & \multicolumn{5}{|c|}{ Mean size of observed reaction } & \multirow{2}{*}{ Mean } \\
\hline & & 0.5hr & 1hr & 24hr & 48hr & 72hr & \\
\hline \multirow{5}{*}{$\begin{array}{c}\text { L1 } \\
\text { crude } \\
\text { extract } \\
(\mathrm{L} 1 \mathrm{CE})\end{array}$} & 0 (control) & $0 \pm 0$ & $0 \pm 0$ & $0 \pm 0$ & $0 \pm 0$ & $0 \pm 0$ & $0 \pm 0$ \\
\hline & 0.5 & $5.23 \pm 1.03$ & $6.33 \pm 0.71$ & $4.4 \pm 0.7$ & $3.93 \pm 0.48$ & $3.2 \pm 0.42$ & $4.62 \pm 0.39$ \\
\hline & 1.0 & $6.07 \pm 1.11$ & $6.87 \pm 0.72$ & $5.07 \pm 0.64$ & $4.57 \pm 0.52$ & $3.77 \pm 0.27$ & $5.27 \pm 0.4$ \\
\hline & 1.5 & $7.53 \pm 1.03$ & $8.3 \pm 0.85$ & $5.97 \pm 0.03$ & $5.63 \pm 0.32$ & $4.67 \pm 0.19$ & $6.42 \pm 0.42$ \\
\hline & Mean & $4.71 \pm 0.94$ & $5.38 \pm 1$ & $3.86 \pm 0.72$ & $3.53 \pm 0.66$ & $2.91 \pm 0.54$ & - \\
\hline \multirow{5}{*}{$\begin{array}{l}\text { Salivary } \\
\text { gland } \\
\text { extract } \\
(\mathrm{SG})\end{array}$} & 0 (control) & $0 \pm 0$ & $0 \pm 0$ & $0 \pm 0$ & $0 \pm 0$ & $0 \pm 0$ & $0 \pm 0$ \\
\hline & 0.5 & $4.17 \pm 0.44$ & $5.2 \pm 0.95$ & $3.37 \pm 0.22$ & $3.13 \pm 0.2$ & $2.97 \pm 0.26$ & $3.77 \pm 0.29$ \\
\hline & 1.0 & $5 \pm 0.45$ & $6.03 \pm 0.72$ & $4.07 \pm 0.34$ & $3.67 \pm 0.39$ & $3.47 \pm 0.28$ & $5 \pm 0.31$ \\
\hline & 1.5 & $5.47 \pm 0.32$ & $6.73 \pm 0.56$ & $4.5 \pm 0.51$ & $4 \pm 0.5$ & $3.63 \pm 0.32$ & $4.87 \pm 0.34$ \\
\hline & Mean & $3.66 \pm 0.67$ & $4.49 \pm 0.85$ & $2.98 \pm 0.55$ & $2.7 \pm 0.5$ & $2.52 \pm 0.46$ & - \\
\hline \multirow{2}{*}{\multicolumn{2}{|c|}{ Mean of antigen type }} & L1CE & SG & - & - & - & - \\
\hline & & $4.08 \pm 0.36^{\mathrm{A}}$ & $3.27 \pm 0.28^{\mathrm{B}}$ & - & - & - & - \\
\hline \multirow{2}{*}{\multicolumn{2}{|c|}{ Mean of concentration }} & 0 (control) & $0.5 \mathrm{mg}$ & $1.0 \mathrm{mg}$ & $1.5 \mathrm{mg}$ & - & - \\
\hline & & $0.0 \pm 0.0^{\mathrm{D}}$ & $4.19 \pm 0.25^{\mathrm{C}}$ & $4.86 \pm 0.26^{\mathrm{B}}$ & $5.64 \pm 0.30^{\mathrm{A}}$ & - & - \\
\hline \multirow{2}{*}{\multicolumn{2}{|c|}{ Mean of time }} & $0.5 \mathrm{hr}$ & $1 \mathrm{hr}$ & $24 \mathrm{hr}$ & $48 \mathrm{hr}$ & $72 \mathrm{hr}$ & - \\
\hline & & $4.18 \pm 0.58^{\mathrm{B}}$ & $4.93 \pm 0.65^{\mathrm{A}}$ & $3.42 \pm 0.45^{\mathrm{C}}$ & $3.12 \pm 0.42^{\mathrm{CD}}$ & $2.71 \pm 0.35^{\mathrm{D}}$ & - \\
\hline
\end{tabular}

a, b \& c: Superscripts to be compared statistically within the same column. Values with different letter superscripts are significantly different $(\mathrm{P}<0.05)$.

A, B \& C: Superscripts to be compared statistically within the same row. Values with different letter superscripts are significantly different $(\mathrm{P}<0.05)$. 
Table (3) Skin reaction measurements (Inch) during different periods by different Rhinoestrus spp. larval antigens concentrations

\begin{tabular}{|c|c|c|c|c|c|c|c|c|c|c|c|c|c|c|c|c|c|}
\hline \multirow{3}{*}{ 氙 } & \multirow{3}{*}{  } & \multicolumn{16}{|c|}{ Antigen type } \\
\hline & & \multicolumn{4}{|c|}{$\begin{array}{c}\text { Excretory-secratory } \\
\text { product(ESP) }\end{array}$} & \multicolumn{4}{|c|}{ Salivary glands extract } & \multicolumn{4}{|c|}{ Mid-gut extract } & \multicolumn{4}{|c|}{ Mixed crude extract } \\
\hline & & $\begin{array}{c}\text { Cont } \\
\text { rol } \\
\text { negat } \\
\text { ive }\end{array}$ & $\begin{array}{l}0.5 \\
\mathrm{mg}\end{array}$ & $1 \mathrm{mg}$ & $\begin{array}{l}1.5 \\
\mathrm{mg}\end{array}$ & $\begin{array}{l}\text { Control } \\
\text { negative }\end{array}$ & $\begin{array}{l}0.5 \\
\mathrm{mg}\end{array}$ & $\begin{array}{c}1 \\
\mathbf{m g}\end{array}$ & $\begin{array}{l}1.5 \\
\mathrm{mg}\end{array}$ & $\begin{array}{c}\text { Contr } \\
\text { ol } \\
\text { negati } \\
\text { ve }\end{array}$ & $\begin{array}{l}0.5 \\
\mathrm{mg}\end{array}$ & $\begin{array}{c}1 \\
\mathbf{m g}\end{array}$ & $\begin{array}{l}1.5 \\
\mathrm{mg}\end{array}$ & $\begin{array}{l}\text { Control } \\
\text { negative }\end{array}$ & $\begin{array}{l}0.5 \\
\mathrm{mg}\end{array}$ & $\begin{array}{c}1 \\
\mathrm{mg}\end{array}$ & $\begin{array}{l}1.5 \\
\mathrm{mg}\end{array}$ \\
\hline \multirow{4}{*}{ 主 } & 1 & 0 & 2 & 4 & 4.5 & 0 & 3 & 3.8 & 3.9 & 0 & 2.5 & 2.9 & 4 & 0 & 2 & 2.1 & 3 \\
\hline & 2 & 0 & 1.7 & 3 & 3.5 & 0 & 2.5 & 3 & 3.6 & 0 & 1.8 & 2.2 & 2.4 & 0 & 1.5 & 3 & 4 \\
\hline & 3 & 0 & 2.7 & 4 & 4.5 & 0 & 2.4 & 2.5 & 2.6 & 0 & 1.5 & 1.7 & 2.1 & 0 & 2.5 & 3 & 3.3 \\
\hline & 1 & 0 & 3.5 & 4.5 & 5.7 & 0 & 5.3 & 5.5 & 6 & 0 & 4.5 & 4.6 & 5.8 & 0 & 2.8 & 5 & 5.5 \\
\hline \multirow[t]{3}{*}{ 크 } & 2 & 0 & 3.6 & 4.6 & 4.8 & 0 & 3.6 & 4.6 & 4.7 & 0 & 1.8 & 2.5 & 3.2 & 0 & 3.5 & 3.9 & 4 \\
\hline & 3 & 0 & 4.8 & 5.1 & 5.6 & 0 & 4 & 4.5 & 5 & 0 & 2.9 & 3.1 & 3.4 & 0 & 3 & 3.5 & 3.5 \\
\hline & 1 & 0 & 3.4 & 4.2 & 5.5 & 0 & 6 & 6.4 & 6.5 & 0 & 2.8 & 3.5 & 4.5 & 0 & 2.8 & 4 & 4.8 \\
\hline \multirow{2}{*}{$\stackrel{\nexists}{\text { 艺 }}$} & 2 & 0 & 3.6 & 4.1 & 4.5 & 0 & 3.8 & 4.9 & 5 & 0 & 3 & 3.5 & 3.5 & 0 & 1.5 & 2.5 & 4 \\
\hline & 3 & 0 & 4.5 & 4.7 & 5 & 0 & 4 & 4.5 & 5 & 0 & 3 & 3.3 & 3.6 & 0 & 1.3 & 1.5 & 2 \\
\hline \multirow{4}{*}{$\underset{+}{\stackrel{\Xi}{+}}$} & 1 & 0 & 1.9 & 2 & 2.5 & 0 & 5.1 & 6.1 & 6.5 & 0 & 2.5 & 2.7 & 4.1 & 0 & 3.6 & 3.7 & 4.5 \\
\hline & 2 & 0 & 2.6 & 3 & 3.2 & 0 & 2 & 3 & 3.4 & 0 & 2.7 & 3 & 0 & 0 & 1.5 & 2.5 & 4 \\
\hline & 3 & 0 & 2.5 & 3.4 & 3.8 & 0 & 3.5 & 3.7 & 4.3 & 0 & 2.2 & 3.3 & 3.6 & 0 & 1.3 & 1.5 & 1.9 \\
\hline & 1 & 0 & 1.9 & 2 & 2.4 & 0 & 4 & 4.8 & 5.9 & 0 & 2 & 2.7 & 4.1 & 0 & 2 & 2.4 & 3.5 \\
\hline \multirow{2}{*}{$\stackrel{\Xi}{\text { ลี }}$} & 2 & 0 & 2.2 & 2.7 & 3.2 & 0 & 1.4 & 2 & 3.4 & 0 & 2.4 & 2.7 & 0 & 0 & 1.5 & 2.5 & 4 \\
\hline & 3 & 0 & 1.7 & 2.5 & 3.6 & 0 & 2.7 & 3 & 4.2 & 0 & 2.2 & 2.7 & 3.4 & 0 & 1.3 & 1.5 & 1.9 \\
\hline
\end{tabular}

-P.I.: post injection.

Table (4) Mean size of skin reaction (Inch) using different Rhinoestrus spp. larval antigen concentrations at different periods of time

\begin{tabular}{|c|c|c|c|c|c|c|c|}
\hline \multirow{2}{*}{ Antigen type } & \multirow{2}{*}{$\begin{array}{c}\text { Concentration } \\
\text { (mg) }\end{array}$} & \multicolumn{5}{|c|}{ Mean size of observed reaction } & \multirow{2}{*}{ Mean } \\
\hline & & $0.5 \mathrm{hr}$ & $1 \mathrm{hr}$ & $24 \mathrm{hr}$ & $48 \mathrm{hr}$ & $72 \mathrm{hr}$ & \\
\hline \multirow{6}{*}{$\begin{array}{l}\text { Excretory } \\
\text { secretory } \\
\text { product } \\
\text { (ESP) }\end{array}$} & 0 (control) & $0 \pm 0^{\mathrm{dA}}$ & $0 \pm 0^{\mathrm{dA}}$ & $0 \pm 0^{\mathrm{dA}}$ & $0 \pm 0^{\mathrm{dA}}$ & $0 \pm 0^{\mathrm{dA}}$ & $0 \pm 0^{\mathrm{d}}$ \\
\hline & 0.5 & $2.13 \pm 0.3^{\mathrm{cBC}}$ & $3.97 \pm 0.42^{\mathrm{cA}}$ & $3.83 \pm 0.34^{\mathrm{cA}}$ & $2.33 \pm 0.22^{\mathrm{cB}}$ & $1.93 \pm 0.15^{\mathrm{cC}}$ & $2.84 \pm 0.26^{\mathrm{c}}$ \\
\hline & 1.0 & $3.67 \pm 0.33^{\mathrm{bC}}$ & $4.73 \pm 0.19^{\mathrm{bA}}$ & $4.33 \pm 0.19^{\mathrm{bB}}$ & $2.80 \pm 0.42^{\mathrm{bD}}$ & $2.40 \pm 0.21^{\mathrm{bE}}$ & $3.59 \pm 0.26^{\mathrm{b}}$ \\
\hline & 1.5 & $4.17 \pm 0.33^{\mathrm{aC}}$ & $5.37 \pm 0.28^{\mathrm{aA}}$ & $5.00 \pm 0.29^{\mathrm{aB}}$ & $3.17 \pm 0.38^{\mathrm{aD}}$ & $3.07 \pm 0.35^{\mathrm{aD}}$ & $4.15 \pm 0.28^{\mathrm{a}}$ \\
\hline & Mean & $2.49 \pm 0.50^{\mathrm{B}}$ & $3.52 \pm 0.64^{\mathrm{A}}$ & $3.29 \pm 0.60^{\mathrm{A}}$ & $2.08 \pm 0.39^{\mathrm{C}}$ & $1.85 \pm 0.36^{\mathrm{C}}$ & - \\
\hline & 0 (control) & $0 \pm 0^{\mathrm{cA}}$ & $0 \pm 0^{\mathrm{dA}}$ & $0 \pm 0^{\mathrm{cA}}$ & $0 \pm 0^{\mathrm{dA}}$ & $0 \pm 0^{\mathrm{dA}}$ & $0 \pm 0^{\mathrm{d}}$ \\
\hline Salivary & 0.5 & $2.63 \pm 0.19^{\mathrm{bC}}$ & $4.30 \pm 0.51^{\mathrm{cA}}$ & $4.60 \pm 0.70^{\mathrm{bA}}$ & $3.53 \pm 0.9^{\mathrm{cB}}$ & $2.70 \pm 0.75^{\mathrm{cC}}$ & $3.55 \pm 0.33^{\mathrm{c}}$ \\
\hline gland extract & 1.0 & $3.10 \pm 0.38^{\mathrm{aD}}$ & $4.87 \pm 0.32^{\mathrm{bB}}$ & $5.27 \pm 0.58^{\mathrm{aA}}$ & $4.27 \pm 0.94^{\mathrm{bC}}$ & $3.27 \pm 0.82^{\mathrm{bD}}$ & $4.15 \pm 0.34^{\mathrm{b}}$ \\
\hline \multirow[t]{3}{*}{ (SG) } & 1.5 & $3.37 \pm 0.39^{\mathrm{aC}}$ & $5.23 \pm 0.39^{\mathrm{aA}}$ & $5.50 \pm 0.50^{\mathrm{aA}}$ & $4.73 \pm 0.92^{\mathrm{aB}}$ & $4.50 \pm 0.74^{\mathrm{aB}}$ & $4.67 \pm 0.31^{\mathrm{a}}$ \\
\hline & Mean & $2.28 \pm 0.42^{\mathrm{D}}$ & $3.60 \pm 0.65^{\mathrm{A}}$ & $3.84 \pm 0.71^{\mathrm{A}}$ & $3.13 \pm 0.66^{\mathrm{B}}$ & $2.62 \pm 0.57^{\mathrm{C}}$ & - \\
\hline & 0 (control) & $0 \pm 0 \mathrm{~d}^{\mathrm{A}}$ & $0 \pm 0^{\mathrm{dA}}$ & $0 \pm 0^{\mathrm{dA}}$ & $0 \pm 0^{\mathrm{cA}}$ & $0 \pm 0^{\mathrm{cA}}$ & $0 \pm 0^{\mathrm{c}}$ \\
\hline Midgut & 0.5 & $1.93 \pm 0.30^{\mathrm{cC}}$ & $3.07 \pm 0.78^{\mathrm{cA}}$ & $2.93 \pm 0.07^{\mathrm{cA}}$ & $2.47 \pm 0.15^{\mathrm{bB}}$ & $2.20 \pm 0.12^{\mathrm{bBC}}$ & $2.52 \pm 0.19^{\mathrm{b}}$ \\
\hline extract & 1.0 & $2.27 \pm 0.35^{\mathrm{bC}}$ & $3.40 \pm 0.62^{\mathrm{bA}}$ & $3.43 \pm 0.07^{\mathrm{bA}}$ & $3.00 \pm 0.17^{\mathrm{aB}}$ & $2.70 \pm 0.10^{\mathrm{aB}}$ & $2.96 \pm 0.17^{\mathrm{a}}$ \\
\hline \multirow{3}{*}{ (MG) } & 1.5 & $2.83 \pm 0.59^{\mathrm{aB}}$ & $4.13 \pm 0.84^{\mathrm{aA}}$ & $3.87 \pm 0.32^{\mathrm{aA}}$ & $2.57 \pm 1.29^{\mathrm{bBC}}$ & $2.50 \pm 1.27^{\mathrm{abC}}$ & $3.18 \pm 0.40^{\mathrm{a}}$ \\
\hline & Mean & $1.76 \pm 0.36^{\mathrm{B}}$ & $2.65 \pm 0.55^{\mathrm{A}}$ & $2.56 \pm 0.46^{\mathrm{A}}$ & $2.01 \pm 0.45^{\mathrm{B}}$ & $1.85 \pm 0.42^{\mathrm{B}}$ & - \\
\hline & 0 (control) & $1.50 \pm 0.12^{\mathrm{dA}}$ & $0.47 \pm 0.03^{\mathrm{cB}}$ & $0 \pm 0^{\mathrm{cC}}$ & $0 \pm 0^{\mathrm{dC}}$ & $0 \pm 0^{\mathrm{dC}}$ & $0.39 \pm 0.16^{\mathrm{d}}$ \\
\hline \multirow{4}{*}{$\begin{array}{l}\text { Mixed crude } \\
\text { extract } \\
(\text { MixCE) }\end{array}$} & 0.5 & $2.00 \pm 0.29^{\mathrm{cB}}$ & $3.10 \pm 0.21^{\mathrm{bA}}$ & $1.87 \pm 0.47^{\mathrm{bB}}$ & $2.13 \pm 0.74^{\mathrm{cB}}$ & $1.60 \pm 0.21^{\mathrm{cC}}$ & $2.14 \pm 0.21^{\mathrm{c}}$ \\
\hline & 1.0 & $2.70 \pm 0.30^{\mathrm{bB}}$ & $4.13 \pm 0.45^{\mathrm{aA}}$ & $2.67 \pm 0.73^{\mathrm{aB}}$ & $2.57 \pm 0.64^{\mathrm{bB}}$ & $2.13 \pm 0.32^{\mathrm{bC}}$ & $2.84 \pm 0.27^{\mathrm{b}}$ \\
\hline & 1.5 & $3.43 \pm 0.30^{\mathrm{aBC}}$ & $4.33 \pm 0.60^{\mathrm{aA}}$ & $3.60 \pm 0.83^{\mathrm{aB}}$ & $3.47 \pm 0.80^{\mathrm{aB}}$ & $3.13 \pm 0.63^{\mathrm{aC}}$ & $3.59 \pm 0.27^{\mathrm{a}}$ \\
\hline & Mean & $2.41 \pm 0.25^{\mathrm{B}}$ & $3.01 \pm 0.49^{\mathrm{A}}$ & $2.03 \pm 0.47^{\mathrm{C}}$ & $2.04 \pm 0.47^{\mathrm{C}}$ & $1.72 \pm 0.38^{\mathrm{C}}$ & - \\
\hline \multirow{2}{*}{\multicolumn{2}{|c|}{ Mean of antigen type }} & ESP & SG & MG & MixCE & - & - \\
\hline & & $2.65 \pm 0.24^{\mathrm{B}}$ & $3.09 \pm 0.27^{\mathrm{A}}$ & $2.17 \pm 0.20^{\mathrm{C}}$ & $2.24 \pm 0.19^{C}$ & - & - \\
\hline \multirow{2}{*}{\multicolumn{2}{|c|}{ Mean of concentration }} & 0 (control) & $0.5 \mathrm{mg}$ & $1.0 \mathrm{mg}$ & $1.5 \mathrm{mg}$ & - & - \\
\hline & & $0.10 \pm 0.04^{\mathrm{D}}$ & $2.76 \pm 0.14^{\mathrm{C}}$ & $3.28 \pm 0.15^{\mathrm{B}}$ & $3.90 \pm 0.17^{\mathrm{A}}$ & - & - \\
\hline \multirow{2}{*}{\multicolumn{2}{|c|}{ Mean of time }} & $0.5 \mathrm{hr}$ & $1 \mathrm{hr}$ & $24 \mathrm{hr}$ & $48 \mathrm{hr}$ & $72 \mathrm{hr}$ & - \\
\hline & & $2.23 \pm 0.20^{\mathrm{B}}$ & $3.19 \pm 0.29^{\mathrm{A}}$ & $2.93 \pm 0.22^{\mathrm{A}}$ & $2.32 \pm 0.29^{\mathrm{B}}$ & $2.01 \pm 0.24^{\mathrm{B}}$ & - \\
\hline
\end{tabular}

a, b \& c: Superscripts to be compared statistically within the same column. Values with different letter superscripts are significantly different $(\mathrm{P}<0.05)$.

A, B \& C: Superscripts to be compared statistically within the same row. Values with different letter superscripts are significantly different $(\mathrm{P}<0.05$ 


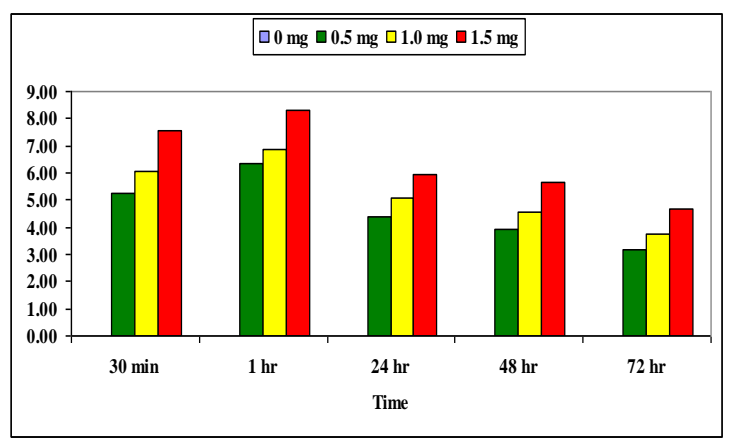

L1 crude extract

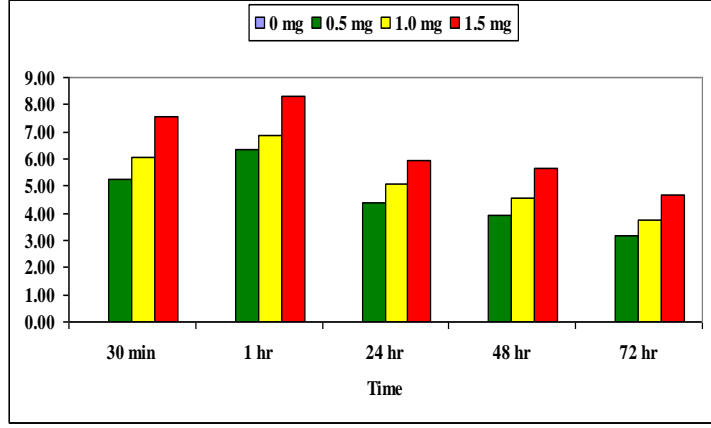

Salivary gland extract

Fig (1) Mean size of skin reaction using different $C$. titillator antigen concentrations at different periods of time

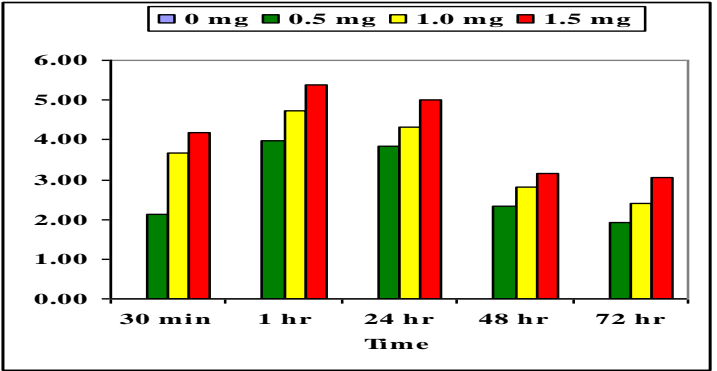

Excretory secretory product



Midgut extract

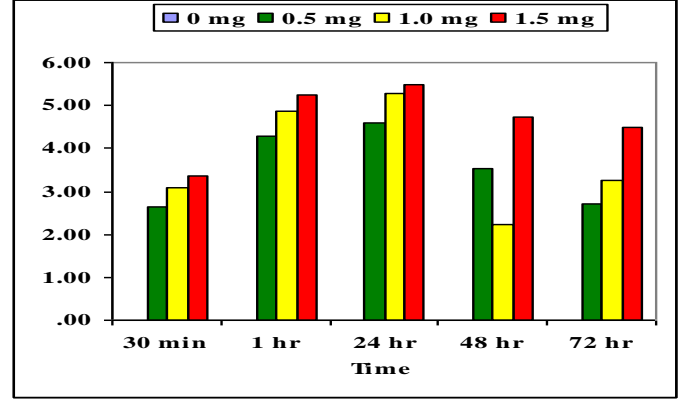

Salivary gland extract

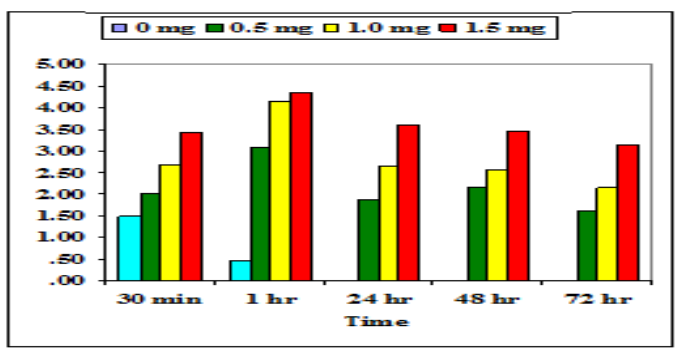

Mixed crude extract

Fig (2) Mean size of skin reaction using different Rhinoestrus spp. antigen concentrations at different periods of

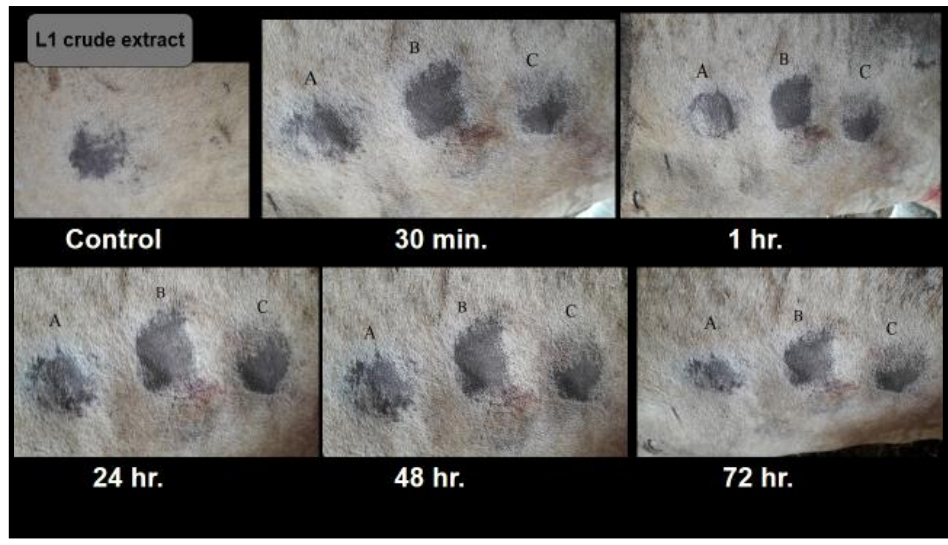

Plate (1) Skin reaction using different Cephalopina titillator L1 crude antigen concentrations at different periods of time
A. $0.5 \mathrm{mg} / \mathrm{ml}$
B. $1 \mathrm{mg} / \mathrm{ml}$
C. $1.5 \mathrm{mg} / \mathrm{ml}$ 


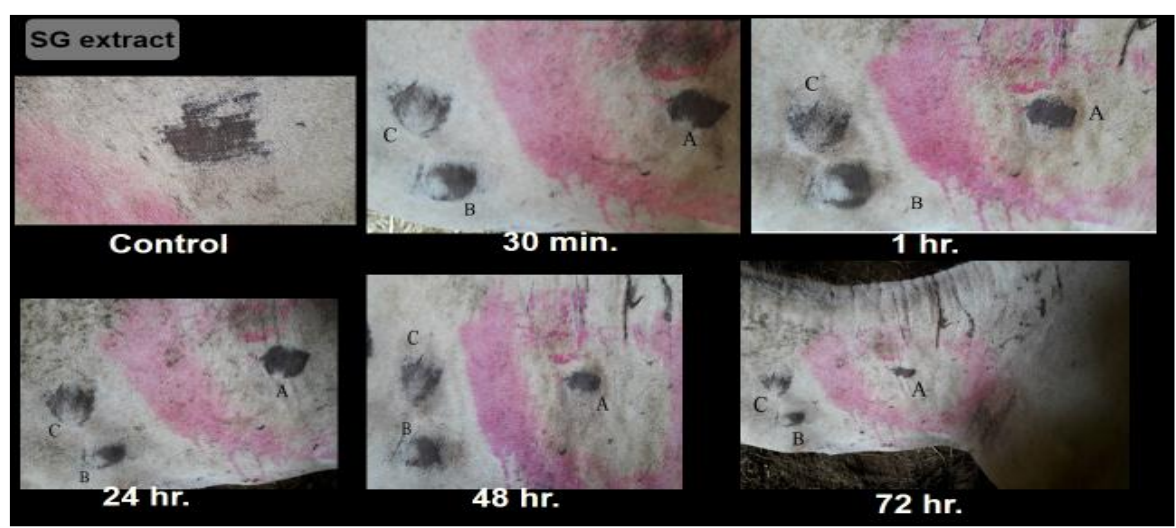

Plate (2) Skin reaction using different Cephalopina titillator Salivary gland antigen concentrations at different periods of time
A. $0.5 \mathrm{mg} / \mathrm{ml}$
B. $1 \mathrm{mg} / \mathrm{ml}$
C. $1.5 \mathrm{mg} / \mathrm{ml}$

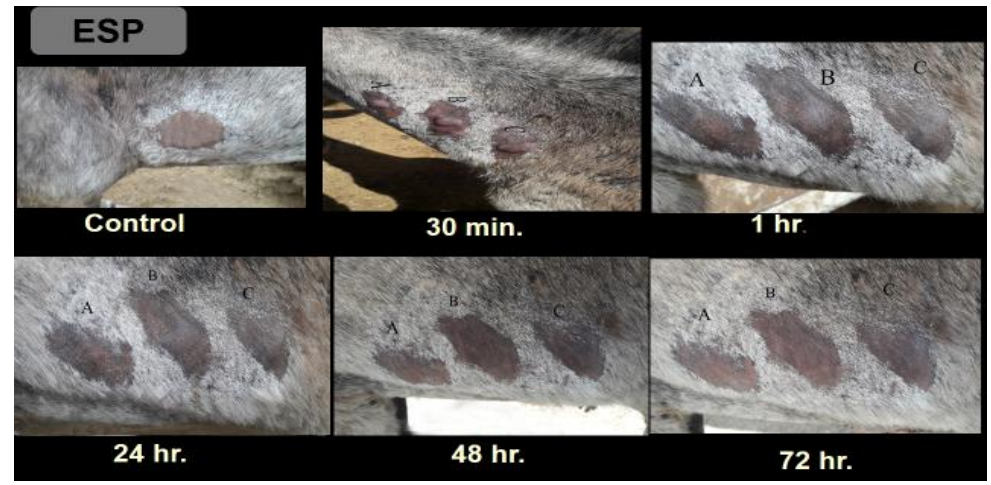

Plate (3)Skin reaction using different Rhinoestrus spp. ESP antigen concentrations at different periods of time A. $0.5 \mathrm{mg} / \mathrm{ml}$

B. $1 \mathrm{mg} / \mathrm{ml}$

C. $1.5 \mathrm{mg} / \mathrm{ml}$



Plate (4) Skin reaction using different Rhinoestrus spp. Salivary gland antigen concentrations at different periods of time
A. $0.5 \mathrm{mg} / \mathrm{ml}$
B. $1 \mathrm{mg} / \mathrm{ml}$
C. $1.5 \mathrm{mg} / \mathrm{ml}$ 


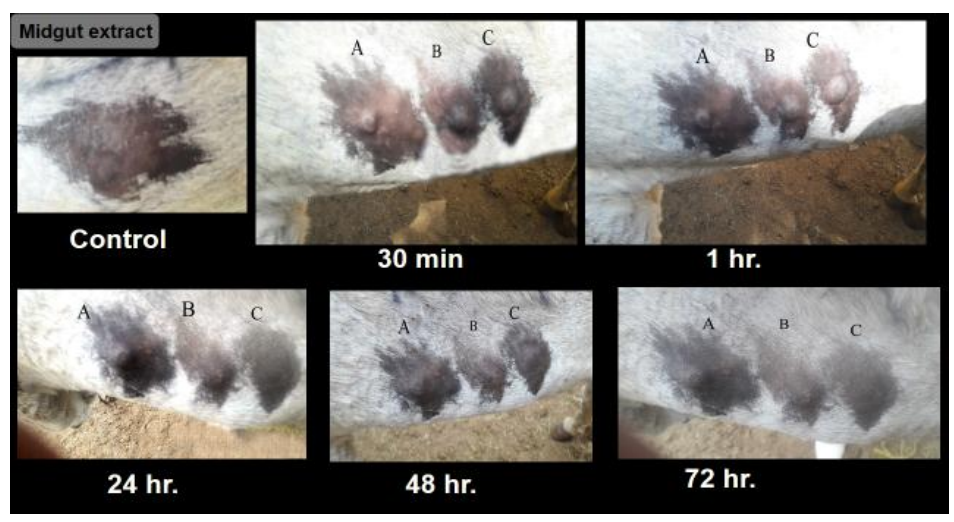

Plate (5) Skin reaction using different Rhinoestrus spp. Midgut antigen concentrations at different periods of time
A. $0.5 \mathrm{mg} / \mathrm{ml}$
B. $1 \mathrm{mg} / \mathrm{ml}$
C. $1.5 \mathrm{mg} / \mathrm{ml}$



Plate (6)Skin reaction using different Rhinoestrus spp. Mixed crude antigen concentrations at different periods of time
A. $0.5 \mathrm{mg} / \mathrm{ml}$
B. $1 \mathrm{mg} / \mathrm{ml}$
C. $1.5 \mathrm{mg} / \mathrm{ml}$

\section{References}

[1] R.Al-Khateeb, Biological Studies on Cephalopina titillator infesting camels in Egypt, MSc Thesis, Cairo University, p.98, 1992.

[2] C.E. Angulo-Valadez, P.J. Scholl, R. CepedaPalacios, P.Jacquiet, P.Dorchies, Nasal bots... a fascinating world!, Veterinary parasitology, Vol.174(1), pp.19-25, 2010.

[3] K.Ashmawy, M. Fahmy, M. Hilali, Incidence and seasonal variations of the larvae of Cephalopina titillator (Clark, 1797) infesting camels (Camelus dromedarius) in Egypt, Proc. XIIth Sump. of the the Scandinavian Society of Parasitology, Tromso, Norway, pp.17-19, 1985

[4] V.Cozma, C.Cernea, D.Achelăriţei, B. Losson, The pathology of Oestrus ovis and an investigation on the use of a skin hypersensitivity test for the diagnosis of sheep oestrosis, Sci Parasitol, Vol.11, p.35e7, 2010

[5] R.Eid, S.Omar, The major immunogens of Cephalopina titillator and Oestrus ovis third stage larvae in Egypt, Veterinary Medical Journal Giza, Vol.48(2), pp. 239-245, 2000

[6]G.ILCHMANN,T.HIEPE, IMMUNOLOGICAL STUDIES INTO INTRAVITAL DIAGNOSIS OF OESTRIASIS, MONATSHEFTE FUR VETERINARMEDIZIN, Vol.40(9), pp.304307, 1985

[7] P.Jacquiet, T.T.T. Ngoc, X. Nouvel, F. Prevot, C.Grisez, H.T.Yacob, J.-P. Bergeaud, H. Hoste, P. Dorchies, G. Tabouret, Regulation of Oestrus ovis (Diptera: Oestridae) populations in previously exposed and naïve sheep, Veterinary 
immunology and immunopathology, Vol.105(1), pp.95-103, 2005

[8] M.Jagannath, N.Cozab, S.A.Rahman, T.Honnappa, SERODIAGNOSIS OF OESTRUS-OVIS INFESTATION IN SHEEP AND GOATS, Indian Journal of Animal Sciences, Vol.59(10), pp.1220-1224, 1989

[9] Y. Kaboret, P. Deconinck, J. Pangui, J. Akakpo, P. Dorchies, Lesions in Rhinoestrus usbekistanicus (Gan 1947) infection of donkeys (Equus asinus) in Senegal (in French), Revue de Medecine Veterinaire, Vol.148(2), pp.123-126, 1997

[10] D.S.Kettle, Medical and veterinary entomology, Cambridge Univ Press, 1995.

[11] M.Khan, An intradermal test to detect latent warble (Hypoderma spp.) infection in cattle, The Canadian Veterinary Journal, Vol. 22(2), p.36, 1981

[12] K.F.Langner, K.E.Darpel, B.S.Drolet, A.Fischer, S.Hampel, J.E.Heselhaus, P.S.Mellor, P.P.Mertens, W.Leibold, Comparison of cellular and humoral immunoassays for the assessment of summer eczema in horses, Veterinary immunology and immunopathology, Vol.122(1), pp.126-137, 2008

[13] A.Oryan, A.Valinezhad, M.Moraveji, Prevalence and pathology of camel nasal myiasis in eastern areas of Iran, Trop Biomed, Vol.25(1), pp.30-36, 2008

[14] D.Otranto, The immunology of myiasis: parasite survival and host defense strategies, TRENDS in Parasitology, Vol.17(4), pp.176182,2001

[15] J.Pruett, C.Barrett, Induction of intradermal skin reactions in the bovine by fractionated proteins of Hypoderma lineatum, Veterinary parasitology, Vol.16(1-2), pp.137-146, 1984

[16] M.Ramadan, Studies on some ectoparasites of camels, $\mathrm{PhD}$ thesis. Faculty of Veterinary Medicine, Zagazig University, Benha Branch, Egypt, 1997.

[17] R.G.Steel, J.H.Torrie, D.A.Dickey, Principles and procedures of statistics: A biological approach, McGraw-Hill, 1997.

[18] A.Zayed, S.Abdel-Shafy, R El-Khateeb, Surface ultrastructure of posterior abdominal spiracles of third instars of nasal bots of Cephalopina titillator, Oestrus ovis and Rhinoestrus purpureus(Diptera: Oestridae) infesting camels, sheep and donkeys in Egypt, Res J Parasitol , Vol.3, pp. 1-11, 2008

[19] F.Zumpt, Myiasis in Man and Animals in the Old World. A Textbook for Physicians, Veterinarians and Zoologists, Myiasis in Man and Animals in the Old World. A Textbook for Physicians, Veterinarians and Zoologists, 1965. 